\title{
Profound Metabolic Acidosis and Oxoprolinuria in an Adult
}

\author{
Michael J. Hodgman, MD ${ }^{a, c}$, James F. Horn, $M D^{b}$, Christine M. Stork, PharmD ${ }^{c}$, Jeanna M. Marraffa, \\ PharmD $D^{c}$, Michael G. Holland, $M D^{c}$, Richard Cantor, $\mathrm{MD}^{c}$, Patti M. Carmel, NPC ${ }^{b}$
}

aBassett Healthcare, Cooperstown, NY

bCapital Physicians, Albany, NY

cUpstate New York Poison Center, Syracuse, NY

\section{ABSTRACT}

Introduction: Profound metabolic acidosis in critically ill adults sometimes remains unexplained despite extensive evaluation.

Case Report: A 58-year-old female presented in a confused state to the emergency department; she had been confused for several days. Laboratory evaluation revealed a high anion gap metabolic acidosis and modestly elevated acetaminophen level. Lactic acid was only modestly elevated. There was no evidence of ketoacids, salicylate, methanol, or ethylene glycol. A urine sample submitted on day 1 of hospitalization revealed a markedly elevated level of 5-oxoproline.

Discussion: Originally described in children with an inherited defect of glutathione synthetase, 5-Oxoproline is an unusual cause of metabolic acidosis. More recently this disturbance has been recognized in critically ill adults without a recognized inherited metabolic disorder. In most of these cases there has been the concomitant use of acetaminophen. Any causal relationship between acetaminophen and this disturbance is speculative.

Conclusion: In critically ill adults with unexplained metabolic acidosis, 5-Oxoproline should be considered in the differential.

\section{INTRODUCTION}

Profound metabolic acidosis is a medical emergency that requires prompt recognition to ensure acute, appropriate management. Early, potentially life saving therapies may include hemodialysis for a toxic alcohol ingestion, insulin for diabetic ketoacidosis, antibiotics for sepsis, and in some cases drug withdrawal-such as for metformin or nucleoside analogue reverse transcriptase inhibitor associated lactic acidosis. The etiology of metabolic acidosis rarely remains elusive. We are reporting a case of profound metabolic acidosis due to accumulation of the organic acid 5-oxoproline.

\section{Case Report}

A 58-year-old female presented in a confused state to the emergency department of a small community hospital. The patient's husband reported that his wife had been confused for several days. He also reported she had not been eating well, had diminished hearing, and (most recently) rapid breathing; this prompted him to bring the patient to the emergency department.

Her past medical history included analgesic and diazepam abuse, alcoholism, anxiety, depression, an eating disorder, COPD, and migraines. Current medications included fluoxetine, diazepam, lansoprazole, conjugated estrogen, and sumatriptan.

A physical examination revealed a disheveled, cachectic female. She was tachypneic and had difficulty hearing. Blood pressure was $171 / 93 \mathrm{~mm} \mathrm{Hg}$, heart rate was 80 beats per minute, respiratory rate was 35 breaths per minute, and rectal temperature was $32.5^{\circ} \mathrm{C}$. Her pupils were normal and sclera were without icterus. No fruity or other odor was appreciated on her breath. Chest, heart, and abdominal exams were unremarkable. Her extremities were cool and mottled. A neurological exam at presentation did not indicate any focal-motor deficits.

Keywords: metabolic acidosis, acetaminophen, 5-oxoproline

Notes: Funding: There was no outside funding of any kind used for this study. Presented: This study was previously presented as poster: North American Congress of Clinical Toxicology, Seattle, WA, Sept. 2004. J Toxicol Clin Toxicol 2004;42:719 (abstract). Corresponding Author: Michael Hodgman, 250 Harrison St., Suite 201, Syracuse, NY 13202. Email: hodgmanm@upstate.edu 


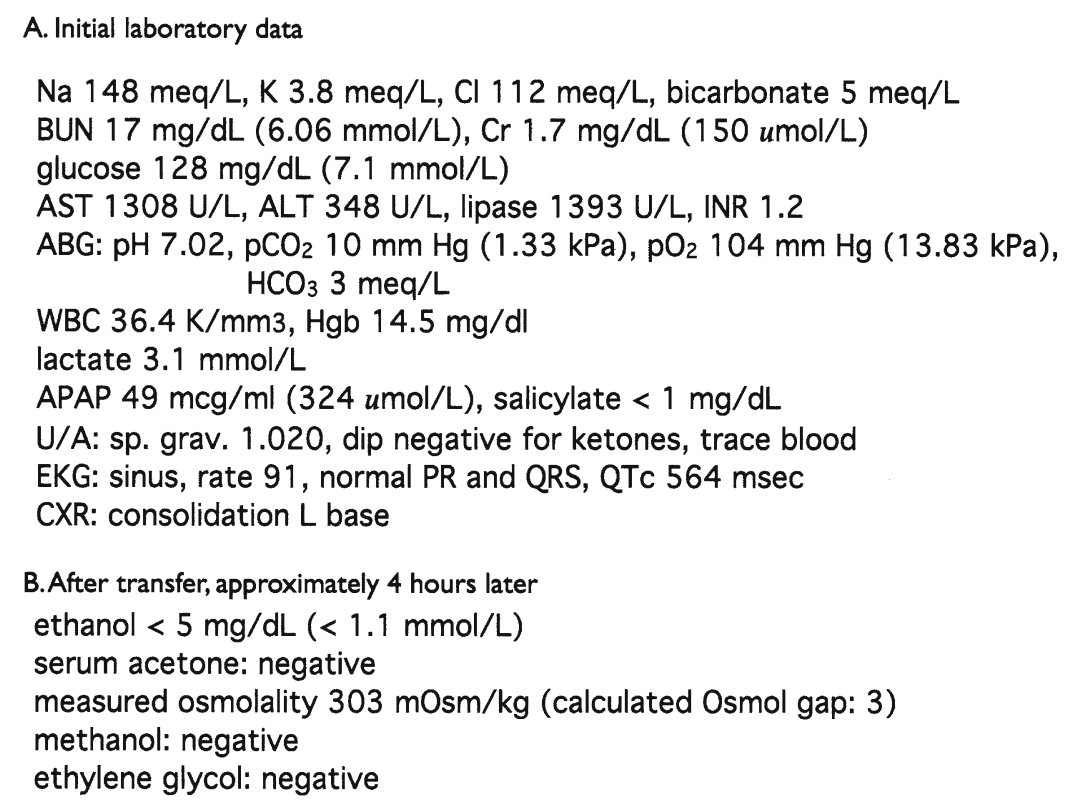

Figure 1: Clinical data on presentation and transfer 4 hours later.

A more complete neurological exam was not performed at that time.

Initial laboratory data revealed a profound metabolic acidosis with an arterial pH of 7.02, pCO2 of $10 \mathrm{~mm} \mathrm{Hg}(1.33 \mathrm{kPa})$, and an anion gap of 31 . Her creatinine was elevated to $1.7 \mathrm{mg} / \mathrm{dL}$ (150 umol/L). Oxygen saturation was calculated at 97\%, and her blood glucose was $128 \mathrm{mg} / \mathrm{dL}(7.1 \mathrm{mmol} / \mathrm{L})$. An electrocardiogram showed a sinus rhythm with a narrow QRS and prolonged QTc to $564 \mathrm{msec}$. A venous lactate was $3.1 \mathrm{mmol} / \mathrm{L}$ and salicylate was negative. She had an AST of $1308 \mathrm{U} / \mathrm{L}$, ALT of $348 \mathrm{U} / \mathrm{L}$, and an acetaminophen level of $49 \mathrm{mcg} / \mathrm{ml}$ (324 umol/L) (Figure 1). Because of the modest elevation of lactic acid, this seemed an unlikely explanation for her profound acidosis and therefore seizure, sepsis, and thiamine deficiency were all considered unlikely. The impression at this point was a potential toxic alcohol ingestion.

Her initial management included active warming with a Bair Hugger ${ }^{\circledR}$ and intravenous fluids with sodium bicarbonate. Because of the elevated acetaminophen level and transaminases with an unknown ingestion history, $\mathrm{N}$-acetyl cysteine was administered orally. She was emergently transferred to a tertiary care facility for further evaluation. Laboratory studies at the tertiary care facility showed a persisting anion gap acidosis with an anion gap of 27 and an arterial $\mathrm{pH}$ of 7.05 with a pCO2 of $15.6 \mathrm{~mm} \mathrm{Hg}$ $(2.07 \mathrm{kPa})$. She was hypokalemic to $2.2 \mathrm{meq} / \mathrm{L}$. Tests for acetone, methanol, and ethylene glycol were all negative.

Her early hospital course was complicated by ventricular arrhythmias that were treated with lidocaine, magnesium, and potassium replacement. N-Acetyl cysteine was continued. Her metabolic acidosis resolved over approximately 48 hours. At discharge, 7 days later, she had a normal creatinine and hepatic transaminases.

A urine organic acid survey submitted on day 1 showed an elevated level of 5-oxoproline, $2350 \mathrm{mmol} / \mathrm{mol}$ creatinine (normal < 100, Quest Diagnostics, Nichols Institute, San Juan, CA).

\section{DISCUSSION}

5-oxoproline is an intermediary of the gamma-glutamyl cycle. This cycle is involved in the transport of amino acids across cell membranes and the synthesis of glutathione (Figures 2,3). Metabolic acidosis associated with 5-oxoproline was first reported in 1970 in a 19-year-old male with a history of mental retardation, spasticity, and gait disturbance [1]. This case led to the recognition of generalized glutathione synthetase deficiency, an inborn metabolic disease that typically presents within the first few days of life with metabolic acidosis, hemolysis, jaundice, and 5-oxoprolinuria. Many patients with this disorder are also disabled by mental retardation and cerebellar or other central nervous system disturbances. The defect has an autosomal recessive pattern of inheritance [2]. In addition to generalized glutathione synthetase deficiency, 5-oxoprolinuria is also reported with other inborn metabolic errors such as urea cycle defects, organic acidurias, and 5-oxoprolinase deficiency [2-4].

Over the last 16 years there have been several case reports and case series of patients with profound metabolic acidosis where extensive investigation has eliminated the more usual causes of metabolic acidosis [5-12]. In these cases large amounts of 5-oxoproline were measured in the urine and in some cases, when measured, in plasma as well. In nearly all cases the acidosis 


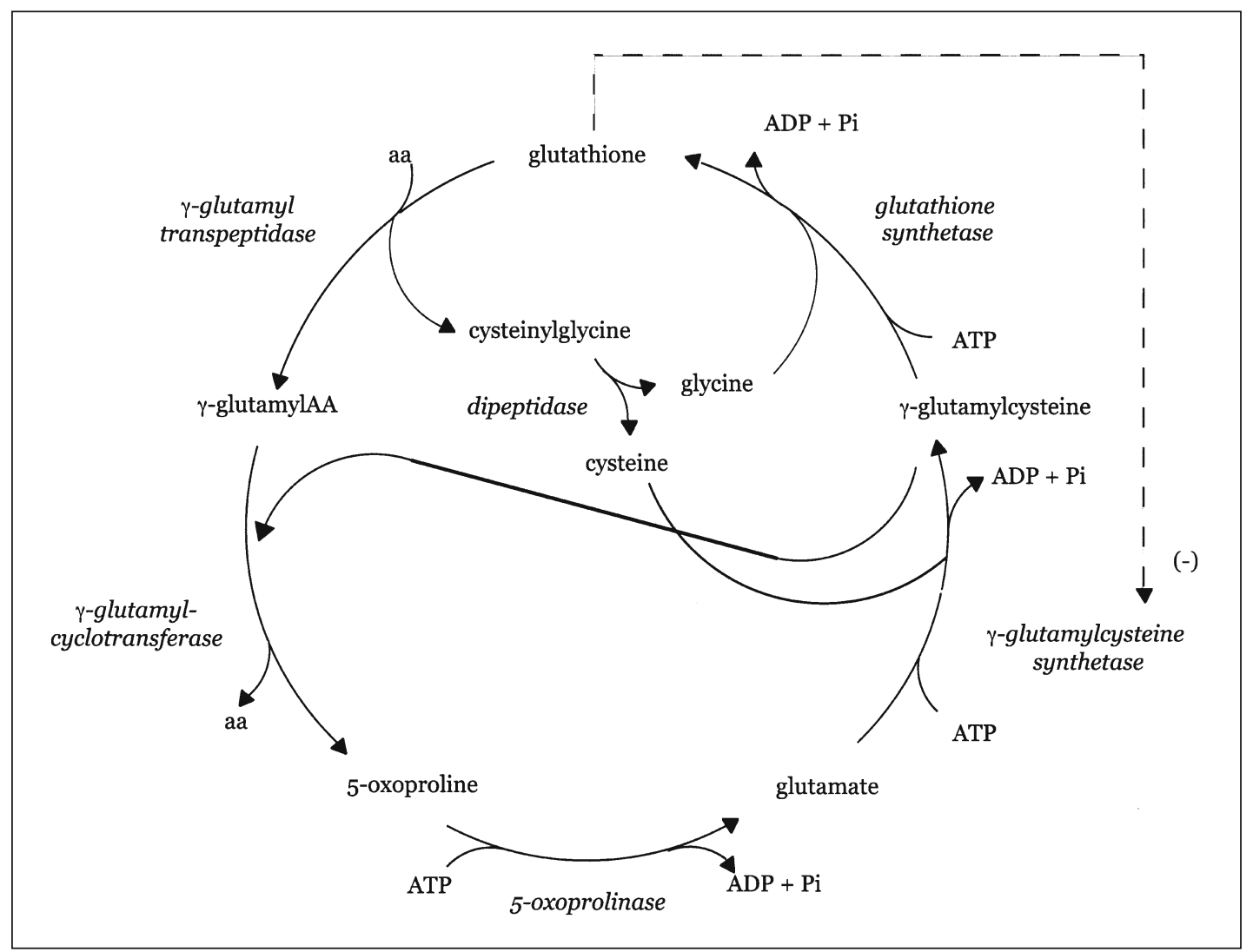

Figure 2: The Gamma-glutamyl cycle

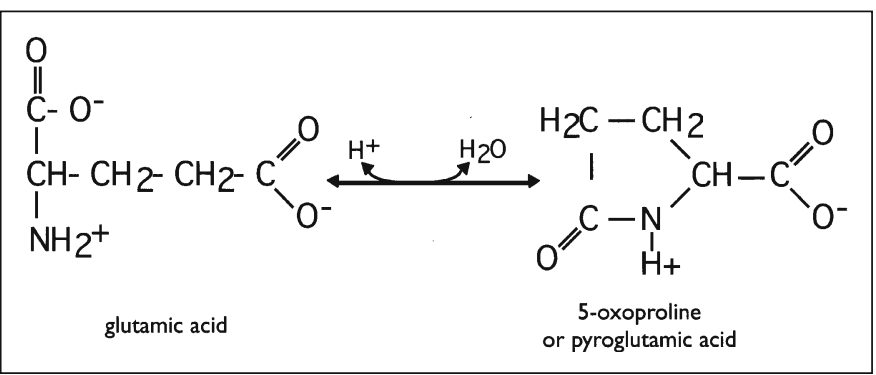

Figure 3: 5-oxoproline

was associated with an acute illness and in most cases the routine use of acetaminophen was reported. None of the cases had a recognized defect of the gamma glutamyl cycle. These cases are outlined in Table 1.

Creer et al. described a 52-year-old female, with end-stage renal disease and renal transplant, who was admitted to a hospital with a high anion gap acidosis after a recent episode of cytomegalovirus colitis. Her serum lactate was normal, and assays for salicylate, ketones, methanol, ethylene glycol, and D-lactate were all negative. Urine qualitative drug screening showed propoxyphene, norpropoxyphene, and acetaminophen. Subsequent testing showed elevated levels of 5-oxoproline in plasma and urine. This patient recovered with supportive measures [5].
Pitt and Brown reported the death of a 34-year-old woman, with "poor eating habits," who was hospitalized with pneumococcal pneumonia, sepsis, and metabolic acidosis. Organic acid analysis revealed elevated 5-oxoproline in her blood and urine, $16 \mathrm{mmol} / \mathrm{L}$ and 13,000 mmol/mol creatinine respectively (nor$\mathrm{mal}<0.07 \mathrm{mmol} / \mathrm{L}$ for blood and $<70 \mathrm{mmol} / \mathrm{mol}$ creatinine for urine). They also reported a "moderate lactic acidosis" and the presence of acetaminophen but did not quantitate these [6].

Pitt and Hauser reviewed 11 additional cases of 5-oxoprolinuria. These cases included 8 adults, a 17-year-old, and 2 children. All were acutely ill, had an anion gap acidosis, and were using acetaminophen. In 3 cases excessive acetaminophen use was suspected. Comorbidities in these 11 cases included pneumonia, pyelonephritis, myxedemic crisis, an infected ventricular peritoneal shunt, subacute bacterial endocarditis, and pancreatitis. The only death occurred in a 64-year-old female with a mixed drug overdose. With treatment of their underlying illness, all the other patients recovered. In the 3 cases with suspected excessive acetaminophen ingestion, $\mathrm{N}$-acetylcysteine was also given [7].

Croal et al. reported the case of a previously healthy 35-yearold female who was hospitalized with a right lower lobe lung abscess [8]. She was receiving flucloxacillin and netilmicin. On day 19 of hospitalization, she had a high anion gap acidosis with an anion gap of $29 \mathrm{mmol} / \mathrm{L}, \mathrm{pH}$ of 7.17 , and a $\mathrm{pCO}_{2}$ of 
Table 1: Summary of other case reports, from references 5-12

\begin{tabular}{|c|c|c|c|c|c|c|}
\hline & & pH & AG & APAP & 5-OP, blood & 5-OP, urine \\
\hline Creer, 1989 & 52 yo female & 7.17 & 27 & yes & 12.6 & $(0.91-1.6 \mathrm{~g} / 24 \mathrm{~h})$ \\
\hline Pitt, 1990 & 34 yo female & 7.17 & NR & yes & 16 & 13,000 \\
\hline \multirow[t]{11}{*}{ Pitt, 1998} & 33 yo female & 7.12 & 33 & yes & ND & 17,000 \\
\hline & 54 yo female & 7.23 & 26 & yes & 6.7 & 13,800 \\
\hline & 60 yo female & 7.14 & 39 & yes & 6.6 & 11,000 \\
\hline & 56 yo female & 7.16 & 31 & yes & 6.6 & 10,000 \\
\hline & 65 yo female & 6.80 & 38 & yes & 8.0 & 4,000 \\
\hline & 6 yo female & 7.49 & 29 & yes & ND & 5,900 \\
\hline & 17 yo female & 7.38 & 31 & yes & ND & 20,400 \\
\hline & 1.2 yo female & 7.29 & 16 & NR & ND & 600 \\
\hline & 73 yo female & 7.31 & 31 & yes & 16 & 22,000 \\
\hline & 83 yo male & 7.15 & 21 & yes & 11.3 & 23,600 \\
\hline & 57 yo male & 7.09 & 37 & yes & 2.3 & 5,700 \\
\hline Croal, 1998 & 35 yo female & 7.17 & 29 & NR & ND & 6,400 \\
\hline \multirow[t]{4}{*}{ Dempsey, 2000} & 80 yo female & 7.27 & 35 & yes & ND & "elevated" \\
\hline & 60 yo female & 7.14 & 38 & yes & 6.6 & 13,700 \\
\hline & 64 yo female & 6.80 & 33 & yes & 8.0 & 4,000 \\
\hline & 54 yo female & 7.26 & 22 & yes & 6.7 & 13,800 \\
\hline Yale, 2000(a) & 44 yo female & NR & 25 & yes & ND & 554 \\
\hline Humphreys, 2005 & 41 yo male & NR & 29 & yes & ND & 5,800 \\
\hline Tailor, 2005(b) & 48 yo male & 7.32 & 26 & yes & ND & 20,700 \\
\hline \multicolumn{7}{|c|}{$\begin{array}{l}p H=\text { arterial } \mathrm{pH}, A G=\text { anion gap, } A P A P=\text { acetaminophen, } 5-\mathrm{OP}=5 \text {-oxoproline, } \mathrm{Cr}=\text { creatin } \\
N R=\text { not reported, } \mathrm{ND}=\text { not done } \\
5-\mathrm{OP} \text { blood: } \mathrm{mmol} / \mathrm{L}, \text { normal }<0.07 \mathrm{mmol} / \mathrm{L} \text {, } \\
5-\mathrm{OP} \text { urine: } \mathrm{mmol} / \mathrm{mol} \mathrm{Cr} \text {, normal }<70 \mathrm{mmol} 5-\mathrm{OP} / \mathrm{mol} \mathrm{Cr} \text { (unless otherwise specified) } \\
\text { (a) urine } 5-\mathrm{OP} \text { was measured } 2 \text { days after admission, when patient was clinically improved } \\
\text { (b) data are for admission \#3, the first admission with 5-OP measured }\end{array}$} \\
\hline
\end{tabular}

$13.5 \mathrm{~mm} \mathrm{Hg}(1.79 \mathrm{kPa})$. Her serum lactate was reported as only mildly increased and ketones and alcohols were negative. On day 21 her antibiotics were discontinued and over the next week her acidosis resolved. Subsequent investigation into the cause of the acidosis revealed dramatically elevated urinary 5-oxoproline. The authors speculate that one of the antibiotics, or the combination of antibiotics, interfered with 5-oxoprolinase. This mechanism for the pathogenesis of the acidosis would not be supported by the observed congenital 5-oxoprolinase deficiency pattern, which is 5-oxoprolinuria without metabolic acidosis [2]. The authors did not comment on the use of acetaminophen.

Dempsey and Lyall reported 4 cases of a high anion gap acidosis associated with 5-oxoprolinemia and 5-oxoprolinuria. All 4 cases involved adult females who were using acetaminophen. With management of their underlying illness, 3 of the 4 females recovered. The fourth died after the resolution of her acidosis [9].

Yale and Mazza reported the case of a 44-year-old female who was chronically using acetaminophen with oxycodone for back pain; the woman had a high anion gap acidosis. Lactate was not elevated, and salicylate and ethanol were negative. Betahydroxybutyrate was elevated at $5.3 \mathrm{meq} / \mathrm{L}$ (normal $<0.3 \mathrm{meq} / \mathrm{L}$ after an overnight fast). With supportive care and the discontinuation of acetaminophen, the acidosis resolved. After she was clinically improved, a urine organic acid assay, done several days after admission, showed an elevated urine 5-oxoproline of $554 \mathrm{mmol} / \mathrm{mol}$ creatinine as well as elevated levels of betahydroxybutyrate and acetoacetate. The authors speculate that this woman's acidosis was a result of 5-oxoprolinuria [10]. The data presented suggests ketoacids also contributed to her acidosis.

Recently, several more reports of this disturbance have been reported. Humphreys et al. reported the case of a 41-year-old male who was admitted for chemotherapy because of polycystic kidney disease and lymphoma. His course was complicated by a neutropenic fever that was treated with broad-spectrum antibiotics and acetaminophen. He developed an increasing anion gap 
acidosis unexplained by lactate or ketoacids. A urine organic acid screen revealed a dramatically elevated 5-oxoproline. Despite aggressive care this patient died from sepsis [11].

Tailor et al. described the case of a 48-year-old male with chronic back pain and repeated hospitalizations for profound metabolic acidosis [12]. The etiology of these episodes remained unclear until elevated urinary levels of 5-oxoproline were measured. (Levels were measured during several hospitalizations). Preceeding 5 of 6 hospitalizations, the patient has used acetaminophen for back pain. During those 5 admissions, the patient responded well to a conservative management of intravenous hydration. On the one occasion when acetaminophen use was not confirmed, the patient had acute cholecystitis. The authors did not report any concurrent acute illness associated with the other 5 admissions that included acetaminophen use.

There are several recent excellent reviews of glutathione homeostasis $[2,13,14]$. Intracellular glutathione is maintained by de novo synthesis via the gamma glutamyl cycle and reduction of glutathione disulfide by NADPH-dependent glutathione disulfide reductase [14]. The key regulatory step in the synthesis of glutathione is gamma glutamylcysteine synthetase, the penultimate enzyme in its synthesis. The activity of this enzyme is influenced by glutathione, which acts as a feedback inhibitor. Conversely, oxidative stress and various cytokines have been identified as agents that increase the activity or expression of this enzyme $[2,13]$. The product of this enzymatic step, gamma glutamylcysteine, is rapidly conjugated with glycine by glutathione synthetase to form glutathione (Figure 3).

An example of this system gone awry is congenital generalized glutathione synthetase deficiency. In this disorder, the lack of feedback inhibition by glutathione results in excessive production of gamma glutamylcysteine. Lacking glutathione synthetase, gamma glutamylcysteine is metabolized by gamma glutamyl cyclotransferase to 5-oxoproline (Figure 3). The excessive production of 5-oxoproline results in acidosis and 5-oxoprolinuria [2].

Increased production of 5-oxoproline during periods of malnourishment or increased caloric demand, such as associated with burns, has been described $[15,16]$. Persaud et al. have found that, during feeding and recovery from severe malnourishment, infants excrete increased urine 5-L-oxoproline. (5-L-oxoproline is the enantiomer endogenously produced by the gamma glutamyl cycle). Glycine supplementation, in most cases, resulted in a decrease in urine 5-L-oxoproline [15]. In infants with the most rapid rate of weight gain, glycine supplementation did not result in a decrease in urine 5-L-oxoproline; this suggests insufficient glycine during the supplementation of these infants. In a study that looked at severely burned adults, whole blood glutathione synthesis was reduced and urine excretion of 5-L-oxoproline increased [16]. The authors of this study speculated that depletion of glutathione precursors led to reduced glutathione synthesis and increased 5-oxoproline excretion.

In our case and the other cited cases, the pathogenesis of the metabolic acidosis is probably multifactorial. Glutathione depletion, malnourishment, critical illness, and perhaps an unrecognized inherited or acquired defect of a critical enzyme (such as glutathione synthetase) are all potentially contributing factors. In the case described by Tailor et al., a measure of the glutathione synthetase activity was taken when the patient was well; it was normal [12].

The use of acetaminophen is reported in most of these cases. A role for acetaminophen in the pathogenesis of this disturbance is intriguing but speculative. In our case it appears that excessive amounts of acetaminophen may have been ingested, but it should be noted that acetaminophen was used therapeutically in most of the other reported cases. Acetaminophen is a safe, universally used analgesic, and its use by these ill patients may be merely coincidental. However, several reports suggest an association $[17,18]$.

Pitt et al. examined random urines, submitted for metabolic screening, for the presence of acetaminophen and 5-oxoproline. Of 10 specimens with acetaminophen, 8 specimens had modest concentrations of 5-oxoproline (10-400 mmol 5-oxoproline/ mol creatinine). Of 10 specimens without acetaminophen, only 1 specimen had 5-oxoproline ( $1 \mathrm{mmol} 5$-oxoproline/ mol $\mathrm{Cr}$ ) [17]. Rats chronically fed a diet high in acetaminophen, about $1 \mathrm{~g} / \mathrm{kg} /$ day, demonstrated elevated urine 5-oxoproline, 50-1000 $\mathrm{mM}$. Neither control rats nor acetaminophen fed rats, whose diet was also supplemented with methionine, excreted excess 5-oxoproline [18]. The dosing in this rat study is certainly excessive and limits any conclusions.

Several recent papers by Stern et al. suggest the kidneys as a potential source of 5-oxoproline $[19,20]$. In mice that receive the acetaminophen metabolite, acetaminophen-cysteine, prior to the administration of acetaminophen, renal glutathione stores are reduced and acetaminophen nephrotoxicity is enhanced [19]. Inhibition of renal tubular brush border gamma-glutamyl transpeptidase prevents the depletion of renal glutathione by this acetaminophen-cysteine adduct [20]. The authors speculate that acetaminophen-cysteine, as a gamma glutamyl transpeptidase substrate, depletes glutathione during the adduct's uptake into renal tubular cells. Depletion of renal glutathione by this mechanism may not only render renal cells more susceptible to injury by renally generated N-acetyl-p-benzoquinoneimine (NAPQI), but it may also result in increased production of 5-oxoproline by renal tubular cells. Although they did not measure 5-oxoproline as part of their study, the results suggest a mechanism for enhanced renal 5-oxoproline production. This mechanism for selective depletion of renal glutathione may also explain the observation of renal injury, without hepatic injury, sometimes seen after acetaminophen poisoning [20].

In summary, we are presenting a case of profound metabolic acidosis in a critically ill female. We performed an exhaustive search for the cause of her acidosis. We did not identify any of the usual causes of metabolic acidosis, but we did identify a large amount of 5-oxoproline in her urine, and we suspect her acidosis was due to 5-oxoprolinemia. With aggressive, supportive care, she made a full recovery. 5-Oxoproline as a cause of severe metabolic acidosis is worthy of further investigation and should be considered in the differential of an unexplained severe metabolic acidosis. 
There are several important limitations to our conclusions. Most importantly, we did not measure plasma 5-oxoproline and are extrapolating from the massive amount found in the urine. The dramatic urinary levels measured in this case are in line with the urinary levels in other reported cases of metabolic acidosis attributed to 5-oxoproline that also included plasma levels $[7,8]$.

Another limitation to our report is that we did not speciate 5-oxoproline. 5-L-oxoproline is a product of the gamma glutamyl cycle, whereas 5-D-oxoproline is derived from D-glutamate. Human sources of D-glutamate include foodstuffs and the turnover of intestinal microflora, whose cell walls are rich in D-glutamate $[21,22]$. D-glutamate is cyclized to 5 -D-oxoproline by a specific enzyme found in renal and hepatic tissue, D-glutamate cyclotranferase. In normal circumstances, the fate of 5-L-oxoproline is a return to L-glutamate by 5-oxoprolinase; 5-D-oxoproline is eliminated renally $[22,23]$. We are unaware of any reports of 5-Doxoproline resulting in metabolic acidosis.

The authors have no potential financial conflicts of interest to report.

\section{REFERENCES}

1. Jellum E, Kluge T, Borresen HC, Stokke O, Eldjarn L. Pyroglutamic aciduria- a new inborn error of metabolism. Scand J Clin Lab Invest. 1970;26:327-335.

2. Larsson A, Anderson M. Glutathione synthetase deficiency and other disorders of the gamma-glutamyl cycle. In: Scriver C, Baudet AL, Sly WS, Valle D, Childs B, Kinzler K, Vogelstein B, eds. The Metabolic and Molecular Basis of Inherited Disease, 8th ed. New York, NY: McGraw-Hill; 2001. pp. 2205-2216.

3. Mayatepek E. 5-Oxoprolinuria in patients with and without defects in the gamma-glutamyl cycle. Eur J Pediatr. 1999; 158:221-225.

4. Larsson A, Mattsson B, Wauters EA, van Gool JD, Duran M, Wadman SK. 5-Oxoprolinuria due to hereditary 5-oxoprolinase deficiency in two brothers- a new inborn error of the gamma-glutamyl cycle. Acta Pediatr Scand. 1981;70:301-308.

5. Creer M, Lau B. Pyroglutamic acidemia in an adult patient. Clin Chem. 1989;35:684-86.

6. Pitt JJ, Brown GK, Clift V, Christodoulou J. Atypical pyroglutamic aciduria: possible role of paracetamol. J Inher Metab Dis. 1990;13:755-56.

7. Pitt J, Hauser S. Transient 5-oxoprolinuria and high anion gap metabolic acidosis; clinical and biochemical findings in eleven subjects. Clin Chem. 1998;44:1497-1503.

8. Croal BL, Glen AC, Kelly CJ, Logan RW. Transient 5-oxoprolinuria (pyroglutamic aciduria) with systemic acidosis in an adult receiving antibiotic therapy. Clin Chem.

1998; 44:336-340.
9. Dempsey G, Lyall H. Pyroglutamic acidemia: a cause of high anion gap metabolic acidosis. Crit Care Med.

2000;28:1803-07.

10. Yale S, Mazza J. Anion gap acidosis associated with acetaminophen (letter). Ann Intern Med. 2000;133:752-3.

11. Humphreys BD, Forman JP, Zandi-Nejad K, Bazari H, Seifter J, Magee CC. Acetaminophen-induced anion gap metabolic acidosis and 5-oxoprolinuria (pyroglutamic aciduria) acquired in hospital. Am J Kidney Dis. 2005;46:143-146.

12. Tailor P, Raman T, Garganta CL, Njalsson R, Carlsson K, Ristoff E, et al. Recurrent high anion gap metabolic acidosis secondary to 5-oxoproline (pyroglutamic acid). Am J Kidney Dis. 2005; 46:E4-E10.

13. Lu SC. Regulation of glutathione synthesis: current concepts and controversies. FASEB J. 1999;13:1169-1183.

14. Griffith OW. Biologic and pharmacologic regulation of mammalian glutathione synthesis. Free Radic Biol Med. 1999;27:922-935.

15. Persaud C, Forrester T, Jackson A. Urinary excretion of 5-L-oxoproline (pyroglutamic acid) is increased during recovery from severe childhood malnutrition and responds to supplemental glycine. J Nutr. 1996;126:2823-2830.

16. Yu YM, Ryan CM, Fei ZW, Lu XM, Castillo 1, Schultz JT, et al. Plasma L-5-oxoproline kinetics and whole blood glutathione synthesis rates in severely burned adult humans. Am J Physiol Endocrinol Metab. 2002;282:E247-E258.

17. Pitt J. Association between paracetamol and pyroglutamic aciduria (letter). Clin Chem. 1990;36:173-174.

18. Ghauri FY, McLean AE, Beales D, Wilson ID, Nicholson JK. Induction of 5-oxoprolinuria in the rat following chronic feeding with $\mathrm{N}$-acetyl 4-aminophenol (paracetamol). Biochem Pharmacol. 1993;46:953-957.

19. Stern ST, Bruno MK, Hennig GE, Horton RA, Roberts JC, Cohen SD. Contribution of acetaminophen-cysteine to acetaminophen nephrotoxicity in CD-1 mice: I. Enhancement of acetaminophen nephrotoxicity by acetaminophen-cysteine. Toxicol Appl Pharmacol. 2005;202:151-159.

20. Stern ST, Bruno MK, Horton RA, Hill DW, Roberts JC, Cohen SD. Contribution of acetaminophen-cysteine to acetaminophen nephrotoxicity II. Possible involvement of the gamma-glutamyl cycle. Toxicol Appl Pharmacol 2005; 202:160-171.

21. Raj D, Langford M, Krueger S, Shelton M, Welbourne T. Regulatory responses to an oral D-glutamate load: formation of D-pyrrolidone carboxylic acid in humans. Am J Physiol Endocrinol Metab. 2001;280:E214-E220.

22. Palekar AG, Tate SS. Accumulation of 5-oxo-L-proline and 5-oxo-D-proline in the blood plasma in end stage renal disease. Biochem Med. 1975;14:339-345.

23. Meister A, Bukenberger MW, Strassburger M. The optically-specific enzymatic cyclization of d-glutamate. Biochem Z. 1963;338:217-229. 